\title{
Bandwidth Allocation in Cellular Networks with Multiple Interferences*
}

\author{
Reuven Bar-Yehuda ${ }^{\dagger}$ \\ reuven@cs.technion.ac.il
}

\author{
Gleb Polevoy ${ }^{\dagger}$ \\ glebp@cs.technion.ac.il
}

\author{
Dror Rawitz $\ddagger$ \\ rawitz@eng.tau.ac.il
}

\begin{abstract}
We study the problem of bandwidth allocation with multiple interferences. In this problem the input consists of a set of users and a set of base stations. Each user has a list of requests, each consisting of a base station, a frequency demand, and a profit that may be gained by scheduling this request. The goal is to find a maximum profit set of user requests $\mathcal{S}$ that satisfies the following conditions: (i) $\mathcal{S}$ contains at most one request per user, (ii) the frequency sets allotted to requests in $\mathcal{S}$ that correspond to the same base station are pairwise non-intersecting, and (iii) the QoS received by any user at any frequency is reasonable according to an interference model. In this paper we consider two variants of bandwidth allocation with multiple interferences. In the first each request specifies a demand that can be satisfied by any subset of frequencies that is large enough. In the second each request specifies a specific frequency interval. Furthermore, we consider two interference models, multiplicative and additive. We show that these problems are extremely hard to approximate if the interferences depend on both the interfered and the interfering base stations. On the other hand, we provide constant factor approximation algorithms for both variants of bandwidth allocation with multiple interferences for the case where the interferences depend only on the interfering base stations. We also consider a restrictive special case that is closely related to the KNAPSACK problem. We show that this special case is NP-hard and that it admits an FPTAS.
\end{abstract}

Keywords: Approximation algorithms, bandwidth allocation, multiple interferences, interval selection, scheduling.

\section{Introduction}

Allocating bandwidth while maintaining a reasonable level of quality of service is an important part of managing a cellular network (see, e.g., $[18,16]$ ). In this paper we focus on the throughput maximization aspect of bandwidth allocation, namely we study the problem of assigning frequencies to base stations according to user requests so as to maximize system profit.

Recently, several studies regarding cell planning of cellular networks have been performed. Roughly speaking, the cell planning problem is to find a minimum cost set of locations for base stations that will be able to satisfy all user demands. (See [2] for a survey on cell planning). A

${ }^{*}$ A preliminary version of this paper was presented at the 6th ACM SIGACT/SIGMOBILE International Workshop on Foundation of Mobile Computing (DIALM-POMC), 2010.

${ }^{\dagger}$ Department of Computer Science, Technion, Haifa 32000, Israel.

${ }^{\ddagger}$ School of Electrical Engineering, Tel-Aviv University, Tel-Aviv 69978, Israel 
client may be covered by several base stations, and in this case the transmission by a base station may be interfered by the other base stations that transmit at the same frequency. Hence, one of the most interesting aspects of the cell planning problem is the interference model. In wideband systems all transmissions are spread over the entire bandwidth, namely the same spectrum is used in every cell, and therefore simultaneous transmissions may cause interferences. In the Orthogonal Frequency Division Multiplexing (OFDM) system transmissions within cells are kept orthogonal due to hopping in the time frequency grid, but inter-cell interferences are still possible, namely base station transmissions may interfere with other cells (see [21, Chapter 4]). Interference is typically modeled by an interference matrix that provides information about the impact of any base station on the transmission of any other base station in areas that are covered by both cells (see [9, Appendix 6B]).

Following Amzallag et al. [3, 1] we consider two interference models for measuring quality of service. In the multiplicative interference model, each pair of base stations $i$ and $k$ have an interference parameter $\sigma(i, k)$ representing the fraction of service that is lost by a user listening to $i$ transmitting at the same frequency as $k$. If several base stations are transmitting at the same frequency $t$, then the remaining service is received by a user listening to base station $i$ at frequency $t$ is $\prod_{k \in B(t) \backslash\{i\}}(1-\sigma(i, k))$, where $B(t)$ denotes and the set of base stations transmitting at $t$. A user is able to listen to base station $i$ at frequency $t$ only if the interference at $t$ is at most $\delta$, i.e., if $\prod_{k \in B(t) \backslash\{i\}}(1-\sigma(i, k)) \geq 1-\delta$. In the additive interference model, each pair of base stations $i$ and $k$ have an interference parameter $w(i, k)$ representing the interference to a user listening to $i$ transmitting at the same frequency as $k$. We have a QoS upper bound on the interference that is normalized to 1 . A user is able to listen to base station $i$ transmitting at frequency $t$ only if the interference at $t$ is at most 1, i.e., if $\sum_{k \in B(t) \backslash\{i\}} w(i, k) \leq 1$.

In this paper we introduce the problem of bandwidth allocation with multiple interferences in which our goal is to maximize the throughput of an existing cellular network. In this problem the input consists of a set of $m$ users, and a set of $n$ base stations. Each user has a list of requests, each consisting of a base station, a frequency demand, and a profit that may be gained by scheduling this request. The goal in this problem is to find a maximum profit set of user requests that satisfies three types of constraints:

User Constraints: The solution contains at most one request per user.

Base Station Constraints: The frequency sets that are assigned to requests that correspond to the same base station are pairwise non-intersecting.

QoS Constraints: The quality of service received by any user at any frequency is reasonable according to some interference model.

We study two variants of bandwidth allocation with multiple interferences. The first is FREQUENCY Allocation with Multiple Interferences (abbreviated, FAMI) where each request simply specifies a demand that can be satisfied by any subset of frequencies that is large enough. The second is the Interval Selection with Multiple Interferences problem (ISMI) where each request specifies a specific frequency interval (i.e., a consecutive set of frequencies). We note that in ISMI user requests may be implicit. (See Section 4.3 for more details.) We consider both FAMI and ISMI with the two interferences models, the multiplicative and the additive.

We note both problems can be defined such that user requests are for sets of time units or time intervals instead of sets of frequencies or frequency intervals. In this case the QoS constraints are per time unit. 
ISMI is an extension of a single machine scheduling problem called WEIGHTED JOB INTERVAL Selection Problem (WJISP). In WJISP the input consists of $n$ weighted jobs, where a job is a set of intervals on the real line (i.e., time intervals). To schedule a job one of its intervals must be selected. A schedule of several jobs contains a set of non-intersecting jobs intervals. The goal is to find a maximum weight schedule. It is not hard to see that when there is only one base station (i.e., $n=1$ ), the QoS constraints no longer matter, and therefore this special case of ISMI is equivalent to WJISP. Spieksma [20] proved that even the unweighted version of WJISP (i.e., JISP) is APX-hard even for the case where there are at most two intervals per job (JISP2). Hence, ISMI is also APX-hard.

ISMI with uniform interferences captures an extension of WJISP in which we are given a set of identical machines, and we have an upper bound on the number of machines that can work simultaneously. This can model an upper bound on the energy consumption or on the size of the crew operating the machines. We note that scheduling jobs on several machines can be modeled by WJISP by concatenating the schedules for the machines along the real line, and specifying the possible intervals for each job accordingly. However, in ISMI we cannot concatenate the schedules for the base stations due to the QoS constraints.

\section{$1.1 \quad$ Our Results}

We show that both FAMI and ISMI are extremely hard to approximate if the interferences depend on both the interfered and the interfering base stations. More specifically, we show that these problems are not approximable within $n^{\varepsilon}$, for some $\varepsilon>0$, unless $\mathrm{P}=\mathrm{NP}$, even for a very restrictive special case in which there is only one frequency, one request per user, one user request per base station and unit profits.

Given this hardness result we consider the case where the interferences depend only on the interfering base stations. We prove that FAMI is strongly NP-hard and provide a 12-approximation algorithm for FAMI and a 7-approximation algorithm for ISMI. (Recall that ISMI is APX-hard even if $n=1$.) Our algorithms are based on the local ratio technique [8,6]. Our approximation ratios improve if all interferences are small. We also note that using the techniques from [6], our algorithm for ISMI can be modified to deal with frequency windows (see Section 4). The resulting approximation ratio is $7+\varepsilon$, for every $\varepsilon>0$.

Finally, we consider the very restrictive special case of FAMI (or ISMI) with additive interferences, where each user has only one request that contains a specific frequency $t_{0}$. We show that this problem is equivalent to an interesting variant of KNAPSACK, we call BACKPACK, where a set of items $S$ is feasible, if the size of $S \backslash\{i\}$ is at most 1, for every $i \in S$. A "real life" application is as follows: we plan to travel, and we would like to choose a set of clothes such that no matter what we wear, the rest of the clothes can be packed in our backpack. We show that this problem is NP-hard and that it admits an FPTAS.

\subsection{Related Work}

Amzallag et al. [3, 1] (see also [4]) studied the cell planning problem and the budgeted cell planning problem, where the goal is to choose locations for base stations whose cost does not exceed a given budget cap, so as to maximize the number of satisfied clients. They considered interferences that depend on both the interfered and the interfering base stations and also on the user. They show that it is NP-hard to approximate the budgeted cell planning problem and present a $\frac{3 e-1}{e-1}$-approximation 
algorithm for a restrictive special case. They also gave an $O(\log W)$-approximation algorithm for the non-interference case of the cell planning problem, where $W$ is the largest capacity over all base stations selected for opening.

Gomes et al. [11] considered frequency assignment to antennas. In this problem the input contains a function that assigns a minimum separation requirement within the frequency spectrum for each pair of antennas, and a function that assigns an interference for each pair of antennas. Gomes et al. proposed a meta-heuristic approach to minimize the total interference.

Klasing et al. [15] studied bandwidth allocation in radio networks with a binary interference model. Given bandwidth demands between the nodes of a capacitated network and an interference graph defined on the edges of the network, they introduced the notion of round, which corresponds to an independent set in the interference graph. They considered the round weighting problem, where a feasible solution is a set of weighted rounds such that (i) the weight of any round is bounded by the capacity of any edge that participate in this round, and (ii) the demands are satisfied by the weighted sum of the rounds. The goal is to compute a minimum weight set of rounds. They proved that round weighting is hard to approximate within $n^{1-\varepsilon}$, for any $\varepsilon>0$, unless $\mathrm{ZPP}=\mathrm{NP}$ and provided (exact or approximation) algorithms for several special cases.

The reader is referred to $[14,18,16]$ for surveys on bandwidth allocation in cellular and wireless systems.

Spieksma [20] proved that JISP2 is APX-hard and gave a simple greedy algorithm that computes 2-approximate solutions for JISP. A 2-approximation ratio via linear programming for WJISP is implicit in Bar-Noy et al. [7]. Bar-Noy et al. [6] presented a unified approach to approximating resource allocation and scheduling problems based on the local ratio technique [8]. Using this framework they designed an approximation algorithm for WJISP with and without time windows. The corresponding approximation ratios are $(2+\varepsilon)$ and 2 , respectively. They also studied a more general version of WJISP where each job has a demand, and a feasible solution may contain intersecting job intervals as long as the total demand at any time $t$ is at most 1 . The approximation ratio they obtained is 5 (or $5+\varepsilon$, if time windows are allowed). Chuzhoy et al. [10] gave a randomized $\frac{e}{e-1}$-approximation algorithm for JISP.

Finally, Knapsack is NP-hard [13] and admits an FPTAS [19, 12].

\subsection{Paper Organization}

The rest of the paper is organized as follows. In Section 2 we provide formal definitions of the interference models and the problems. The approximation algorithm for FAMI is given in Section 3, and our approximation algorithm for ISMI is presented in Section 4. Our hardness results are given in Section 5 and we consider the BACKPACK problem in Section 6.

\section{Preliminaries}

In this section we provide a description of the interference models and a formal definition of the problems we consider in this paper. We also shortly discuss the local ratio technique.

\subsection{Interference Models}

Our interference models are based on the models from [3]. The basic interference we consider is the interferences caused to a user $j$, listening to a base station $i$ using some frequency $t$, by other 
base stations (transmitting to other users).

Multiplicative interference. Each base station $i$ is associated with an interference parameter $\sigma(i) \in[0,1]$ which represents the fraction of service that is lost by a user listening to another base station that is transmitting at the same frequency as $i$. It follows that the remaining service is $1-\sigma(i)$. If several base stations are transmitting at frequency $t$, then the remaining service is received by a user listening to base station $i$ at frequency $t$ is $\prod_{k \in B(t) \backslash\{i\}}(1-\sigma(k))$, where $B(t)$ denotes the set of base stations transmitting at $t$. The overall interference felt by the user listening to $i$ at frequency $t$ is simply $1-\prod_{k \in K(t)}(1-\sigma(k))$. We have an upper bound $\delta$ on the interference that determines the lowest possible quality of service that a user may receive. A user is able to listen to base station $i$ at frequency $t$ only if the interference is at most $\delta$, i.e., if

$$
\prod_{k \in B(t) \backslash\{i\}}(1-\sigma(k)) \geq 1-\delta .
$$

Additive interference. Each base station has an interference parameter $w(i) \geq 0$ that represents the interference to a user listening to another base station that is transmitting at the same frequency as $i$. The overall interference felt by a user listening to a transmission by base station $i$ at frequency $t$ is $\sum_{k \in B(t) \backslash\{i\}} w(k)$. In the additive model, we have a QoS upper bound on the interference that is normalized to 1. A user is able to listen to base station $i$ at frequency $t$ only if the overall interference at $t$ is at most 1 , i.e., if

$$
\sum_{k \in B(t) \backslash\{i\}} w(k) \leq 1
$$

Amzallag et al. [3] used the additive interference model as a first order approximation of the multiplicative model for very small interferences:

$$
\prod_{k \in B(t) \backslash\{i\}}(1-\sigma(k)) \approx 1-\sum_{k \in B(t) \backslash\{i\}} \sigma(k) .
$$

Another justification for the additive model is obtained by linearization of the multiplicative model. By taking the logarithm of Eqn. (1) we get

$$
\sum_{k \in B(t)} \log (1-\sigma(k)) \geq \log (1-\delta) .
$$

Hence, Eqn. (1) can be restated in linear form, where $w(k) \triangleq \frac{\log (1-\sigma(k))}{\log (1-\delta)}$. We note that both linearizations may cause accuracy problems, namely a feasible solution in the additive model may be infeasible in the multiplicative model and vice versa due to "rounding" of additive interferences.

\subsection{The Problems}

We define the Frequency Allocation with Multiple Interferences problem (FAMi) and the Interval Selection with Multiple Interferences problem (ISMI) for the multiplicative model. Similar definitions can be stated for the additive model.

In FAMI the input consists of a set $U$ of $m$ users, a set $B$ of $n$ base stations, and a frequency cap $f$. Each base station $i \in B$ has an interference parameter $\sigma(i)$ (or $w(i)$ ). Each user $j \in U$ has $n$ sets 
$\mathcal{R}_{j 1}, \ldots, \mathcal{R}_{j n}$ of requests from base stations, where $\mathcal{R}_{j i}$ correspond to base station $i \in B$. Denote $\mathcal{R}=\bigcup_{j i} \mathcal{R}_{j i}$. A request $R_{j i q} \in \mathcal{R}_{j i}$ is associated with a bandwidth demand $d\left(R_{j i q}\right) \in\{1, \ldots, f\}$ and a profit $p\left(R_{j i q}\right)$.

A solution is a pair $(\mathcal{S}, \mathcal{A})$, where $\mathcal{S}$ is a set of requests, and $\mathcal{A}: S \rightarrow 2^{\{1, \ldots, f\}}$ is an assignment of frequencies to the requests in $\mathcal{S}$. Given a solution $(\mathcal{S}, \mathcal{A})$ and a frequency $t$, denote by $\mathcal{S}(t)$ the set of requests to which $t$ is assigned, i.e., $\mathcal{S}(t) \triangleq\{R \in \mathcal{S}: t \in \mathcal{A}(R)\}$. A feasible solution $(\mathcal{S}, \mathcal{A})$ satisfies the following constraints:

User Constraints: At most one request per user is satisfied, i.e., $\left|\bigcup_{i} \mathcal{R}_{j i} \cap \mathcal{S}\right| \leq 1$, for every user $j$.

Demand Constraints: Each request $R \in \mathcal{S}$ is assigned to exactly $d(R)$ frequencies, i.e., $|\mathcal{A}(R)|=$ $d(R)$, for every $R \in \mathcal{S}$.

Base Station Constraints: Two requests $R_{j i q}, R_{j^{\prime} i q^{\prime}} \in \mathcal{S}$ that correspond to the same base station $i$ cannot be assigned to the same frequency, namely $\mathcal{A}\left(R_{j i q}\right) \cap \mathcal{A}\left(R_{j^{\prime} i q^{\prime}}\right)=\emptyset$.

QoS Constraints: The quality of service received by any user $j$ at any frequency $t$ is at least $1-\delta$, i.e.,

$$
\prod_{k \in B(t) \backslash\{i\}}(1-\sigma(k)) \geq 1-\delta,
$$

for every $t$ and $i \in B(t)$, where $B(t)$ is the set of base stations to which frequency $t$ was assigned, namely $B(t)=\left\{i: \exists R_{j i q} \in \mathcal{S}(t)\right\}$.

The goal in FAMI is to find a maximum profit feasible solution.

ISMI is a variant of FAMI in which user requests are for specific frequency intervals. The main difference between the problems is that a request $I_{j i q} \in \mathcal{R}_{j i}$ is not a bandwidth request but rather a frequency interval that is contained in $\{1, \ldots, f\}$. Since we are dealing with intervals, a solution for ISMI can be described using the selected set of requests $\mathcal{S}$, and there is no need for the assignment $\mathcal{A}$ or for the demand constraints. User constraints remain the same, but we rephrase base station and QoS constraints:

Base Station Constraints: The requests in $\mathcal{S}$ that correspond to a base station $i$ are pairwise non-intersecting, namely if $I_{j i q}, I_{j^{\prime} i q^{\prime}} \in \mathcal{S}$, then $I_{i j q} \cap I_{j^{\prime} i q^{\prime}}=\emptyset$, for every base station $i$.

QoS Constraints: The quality of service received by any user $j$ at any frequency $t$ is at least $1-\delta$, i.e.,

$$
\prod_{k \in B(t) \backslash\{i\}}(1-\sigma(k)) \geq 1-\delta,
$$

for every frequency $t$ and base station $i \in B(t)$, where $B(t)$ is the set of base stations that transmit at frequency $t$, namely $B(t)=\left\{i: \exists j \in U\right.$, s.t. $\left.t \in I \in \mathcal{R}_{j i} \cap \mathcal{S}\right\}$.

\subsection{The Local Ratio Technique}

The local ratio technique $[8,6]$ is based on the Local Ratio Theorem, which applies to optimization problems of the following type. The input is a non-negative profit (or penalty) vector $p \in \mathbb{Q}^{n}$ and a set of feasibility constraints $\mathcal{F}$. The problem is to find a solution vector $x \in \mathbb{Q}^{n}$ that maximizes (or minimizes) the inner product $p \cdot x$ subject to the constraints $\mathcal{F}$. 
Theorem 1 (Local Ratio [6]). Let $\mathcal{F}$ be a set of constraints and let $p, p_{1}$, and $p_{2}$ be profit functions such that $p=p_{1}+p_{2}$. Then, if $x$ is $r$-approximate both with respect to $\left(\mathcal{F}, p_{1}\right)$ and with respect to $\left(\mathcal{F}, p_{2}\right)$, for some $r$, then $x$ is also an $r$-approximate solution with respect to $(\mathcal{F}, p)$.

Since we consider linear profit functions, we denote $p(\mathcal{S})=\sum_{R \in \mathcal{S}} p(R)$, for any subset of requests $\mathcal{S} \subseteq \mathcal{R}$. We also abuse notation and denote $w(\mathcal{S})=\sum_{R \in \mathcal{S}} w(R)$, where $w(R)$ is the additive interference of the base station associated with $R$.

\section{$3 \quad$ Frequency Allocation with Multiple Interferences}

In this section we present an algorithm for FAMI that computes 12-approximate solutions. We start with the additive interference model. For $\rho \in\left(0, \frac{1}{2}\right]$, we present a local ratio $\left(5+\frac{2}{1 / \rho-1}\right)$ approximation algorithm for the case where all interferences are at most $\rho$. Next, using similar techniques we design a $(2\lceil 1 / \rho\rceil+1)$-approximation algorithm for the case where all interferences are greater than $\rho$. By combining both algorithms for $\rho=\frac{1}{2}$ we obtain a 12-approximation algorithm for the general case. Our approximation algorithm can also be used for FAMI with the multiplicative interference model. In this case, the $\left(5+\frac{2}{1 / \rho-1}\right)$ ratio is obtained when $\sigma(i) \leq 1-(1-\delta)^{\rho}$, for every base station $i \in B$.

\subsection{Small Interferences}

We first present a $\left(5+\frac{2}{1 / \rho-1}\right)$-approximation algorithm for the special case of FAMI, where all interferences are at most $\rho$, i.e., for the case where $w(i) \leq \rho$, for every base station $i$.

Before presenting our algorithm we need several definitions. Let $Q$ be a request for base station $i$ made by user $j$, namely $Q \in \mathcal{R}_{j i}$. Also, let $\mathcal{U}(Q) \subseteq \mathcal{R}$ denote the set of requests made by user $j$ and let $\mathcal{B}(Q) \subseteq \mathcal{R}$ denote the set of requests from base station $i$ that were not made by user $j$, i.e., $\mathcal{U}(Q) \triangleq \bigcup_{i^{\prime}} \mathcal{R}_{j i^{\prime}}$ and $\mathcal{B}(Q) \triangleq \bigcup_{j^{\prime} \neq j} \mathcal{R}_{j^{\prime} i}$.

Algorithm FA-Small (Algorithm 1) is our local ratio algorithm for FAMI with small interferences. It is recursive and works as follows. If there are no requests, then it returns an empty schedule. Otherwise, it chooses a request $Q$ with minimum demand. It constructs a new profit function $p_{1}$, and solves the problem recursively on $p_{2}=p-p_{1}$ and the set of requests with positive profit with respect to $p_{2}$ that is denoted by $\mathcal{R}^{+}$. Notice that $Q \notin \mathcal{R}^{+}$, since $p_{2}(Q)=0$. Then, it tries to add $Q$ to the solution that was computed recursively, $\left(\mathcal{S}^{+}, \mathcal{A}^{+}\right)$, if possible. This is done using Subroutine Augment that greedily checks whether there are $d(Q)$ frequencies that can be assigned to $Q$.

Observe that the computed solution $(\mathcal{S}, \mathcal{A})$ is feasible by construction. As for the running time, first observe that the number of recursive calls is at most $|\mathcal{R}|$, since each recursive call removes at least one request. In a naive implementation each iteration requires $O(|\mathcal{R}|$ ) operations (finding a request with minimum demand, subtracting profits, and computing $\mathcal{R}^{+}$). Also, observe that checking whether a frequency $t$ can be assigned to the request $Q$ can be done in $O(1)$, since it is enough to check the interference felt by (i) the user requesting $Q$, and (ii) a user whose request corresponds to a base station with minimum interference among the requests to which $t$ was assigned. It follows that the Augment operation can be performed in $O(f)$. Thus the overall complexity of the algorithm is $O\left(|\mathcal{R}|^{2}+|\mathcal{R}| f\right)$. Note that in FAMI the size of the output may be linear in $f$, and therefore the running time of an algorithm for FAMI must be $\Omega(f)$. 


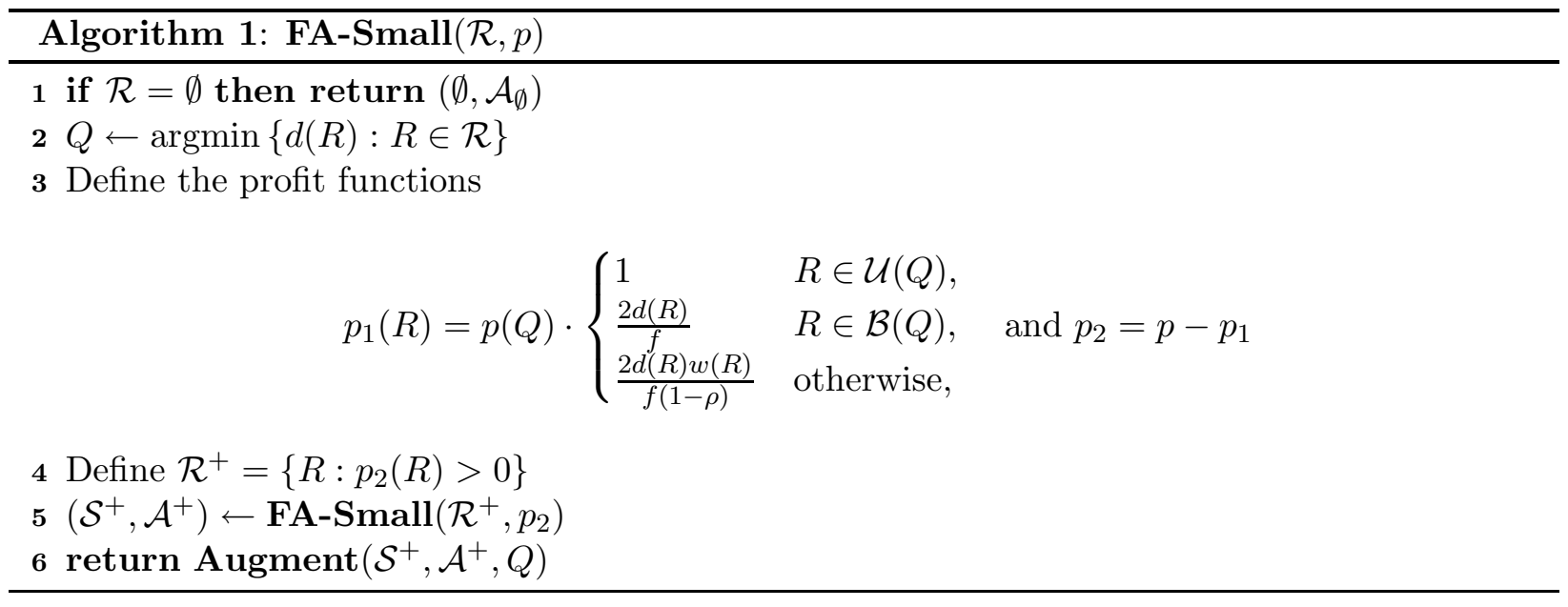

Next, we prove that Algorithm FA-Small computes $\left(5+\frac{2}{1 / \rho-1}\right)$-approximate solutions using the Local Ratio Theorem. To use the theorem we prove that the computed solution is $\left(5+\frac{2}{1 / \rho-1}\right)$ approximate with respect to the profit function $p_{1}$ that is defined in Line 3 . We start by showing that the profit of any feasible solution is at most $\left(5+\frac{2}{1 / \rho-1}\right) p_{1}(Q)$ with respect to $p_{1}$.

Lemma 1. If $\rho \leq \frac{1}{2}$, then $\operatorname{OPT}\left(p_{1}\right) \leq\left(5+\frac{2}{1 / \rho-1}\right) p_{1}(Q)$.

Proof. Let $i$ and $j$ be the base station and user that correspond to $Q$. Let $\left(\mathcal{S}^{\prime}, \mathcal{A}^{\prime}\right)$ be some feasible solution. Since $\mathcal{S}^{\prime}$ may contain at most one request from $\mathcal{U}(Q)$ (user constraint), it follows that $p_{1}\left(\mathcal{S}^{\prime} \cap \mathcal{U}(Q)\right) \leq p_{1}(Q)$. It remains to bound $p_{1}\left(\mathcal{S}^{\prime} \backslash \mathcal{U}(Q)\right)$. To do that we divide the profit $p_{1}(R)$ equally among the frequencies in $\mathcal{A}^{\prime}(R)$, for every request $R \in \mathcal{S}^{\prime} \backslash \mathcal{U}(Q)$, i.e., we assign $p_{1}^{t}(R) \triangleq \frac{p_{1}(R)}{d(R)}$ to each $t \in \mathcal{A}^{\prime}(R)$. Consider a frequency $t$ and notice that $w(\mathcal{S}(t)) \leq 1+\rho$. (Recall that $\mathcal{S}(t)$ is set of requests to which $t$ is assigned.) If there exists $Q^{\prime} \in \mathcal{B}(Q)$ such that $t \in \mathcal{A}\left(Q^{\prime}\right)$, then $w\left(\mathcal{S}^{\prime}(t) \backslash\left\{Q^{\prime}\right\}\right) \leq 1$. It follows that

$$
\begin{aligned}
p_{1}^{t}\left(\mathcal{S}^{\prime}(t) \backslash \mathcal{U}(Q)\right) & =p_{1}^{t}\left(Q^{\prime}\right)+p_{1}^{t}\left(\mathcal{S}^{\prime}(t) \backslash\left(\mathcal{U}(Q) \cup\left\{Q^{\prime}\right\}\right)\right) \\
& =\frac{2 p_{1}(Q)}{f}+\frac{2 p_{1}(Q)}{f(1-\rho)} \cdot w\left(\mathcal{S}^{\prime}(t) \backslash\left(\mathcal{U}(Q) \cup\left\{Q^{\prime}\right\}\right)\right. \\
& \leq \frac{2 p_{1}(Q)}{f}+\frac{2 p_{1}(Q)}{f(1-\rho)} \\
& =\frac{2 p_{1}(Q)}{f} \cdot \frac{2-\rho}{1-\rho} .
\end{aligned}
$$

Otherwise

$$
p_{1}^{t}\left(\mathcal{S}^{\prime}(t) \backslash \mathcal{U}(Q)\right)=\frac{2 p_{1}(Q)}{f(1-\rho)} \cdot w\left(\mathcal{S}^{\prime}(t)\right) \leq \frac{2 p_{1}(Q)}{f} \cdot \frac{1+\rho}{1-\rho} .
$$

Since $\rho \leq \frac{1}{2}$, we have that

$$
p_{1}\left(\mathcal{S}^{\prime}\right)=p_{1}\left(\mathcal{S}^{\prime} \cap \mathcal{U}(Q)\right)+p_{1}\left(\mathcal{S}^{\prime} \backslash \mathcal{U}(Q)\right) \leq p_{1}(Q)+2 p_{1}(Q) \cdot \frac{2-\rho}{1-\rho}=\left(5+\frac{2 \rho}{1-\rho}\right) p_{1}(Q)
$$

and the lemma follows. 
Next, we show that the profit of the computed solution $\mathcal{S}$ is at least $p_{1}(Q)$ with respect to $p_{1}$.

Lemma 2. $p_{1}(\mathcal{S}) \geq p_{1}(Q)$.

Proof. First assume that $d(Q) \leq \frac{1}{2} f$. If $\mathcal{S} \cap \mathcal{U}(Q) \neq \emptyset$, then $p_{1}(\mathcal{S}) \geq p_{1}(Q)$. Otherwise, due to Line 6 we know that $\mathcal{S} \cup\{Q\}$ is infeasible. In this case $Q$ was rejected since there were less than $d(Q)$ available frequencies. A frequency $t$ may be unavailable due to two reasons, either $t \in \mathcal{A}\left(Q^{\prime}\right)$ for some $Q^{\prime} \in \mathcal{B}(Q)$, or due to an interference constraint. As in Lemma 1, we divide the profit $p_{1}(R)$ equally among the frequencies in $\mathcal{A}(R)$, for every request $R \in \mathcal{S}^{\prime} \backslash \mathcal{U}(Q)$. In the former case we have $p_{1}^{t}(\mathcal{S}) \geq \frac{2}{f} \cdot p_{1}(Q)$. In the latter case, $w\left(\mathcal{S}^{\prime}(t) \backslash \mathcal{U}(Q)\right)>1-\rho$ and therefore $p_{1}^{t}(\mathcal{S}) \geq \frac{2}{f} \cdot p_{1}(Q)$. Since $d(Q) \leq \frac{1}{2} f$, it follows that

$$
p_{1}(\mathcal{S})=\sum_{t} p_{1}^{t}\left(S_{Q}(t)\right)>\frac{2 p_{1}(Q)}{f} \cdot(f-d(Q)) \geq p_{1}(Q) .
$$

If $d(Q)>\frac{1}{2} f$, observe that $d(R)>\frac{1}{2} f$ for every $R \in \mathcal{R}$. If $\mathcal{S}$ contains a request from $\mathcal{U}(Q) \cup$ $\mathcal{B}(Q)$, then $p_{1}(\mathcal{S}) \geq p_{1}(Q)$, and we are done. Otherwise, $Q$ is not added to $\mathcal{S}$ due to interference constraints. Let $t$ be a frequency that is unavailable for $Q$. Hence, $w(\mathcal{S}(t))>1-\rho$. It follows that

$$
p_{1}(\mathcal{S}) \geq \sum_{R \in \mathcal{S}(t)} p_{1}(R) \geq p_{1}(Q) \sum_{R \in \mathcal{S}(t)} \frac{2 d(R) w(R)}{f(1-\rho)} \geq p_{1}(Q) \sum_{R \in \mathcal{S}(t)} \frac{f w(R)}{f(1-\rho)}>p_{1}(Q)
$$

as required.

Using Lemmas 1 and 2 and the Local Ratio Theorem we obtain the following result.

Theorem 2. Let $\rho \leq \frac{1}{2}$. Algorithm $\boldsymbol{F A}$-Small is a polynomial time $\left(5+\frac{2}{1 / \rho-1}\right)$-approximation algorithm for the case where $w(i) \leq \rho$, for every base station $i$.

Proof. The proof is by induction on the recursion. At the induction base (Line 1) $\mathcal{R}=\emptyset$ and the algorithm returns an optimal solution. For the induction step, we assume that $\mathcal{S}^{+}$is $\left(5+\frac{2}{1 / \rho-1}\right)$ approximate with respect to $\left(\mathcal{R}^{+}, p_{2}\right)$ and show that $\mathcal{S}$ is $\left(5+\frac{2}{1 / \rho-1}\right)$-approximate with respect to $\left(\mathcal{R}, p_{2}\right)$ and $\left(\mathcal{R}, p_{1}\right)$. This proves, by Local Ratio Theorem, that $\mathcal{S}$ is $\left(5+\frac{2}{1 / \rho-1}\right)$-approximate with respect to $(\mathcal{R}, p)$. First, $\mathcal{S}$ is $\left(5+\frac{2}{1 / \rho-1}\right)$-approximate with respect to $\left(\mathcal{R}, p_{1}\right)$ due to Lemmas 1 and 2. The set $\mathcal{R} \backslash \mathcal{R}^{+}$contains requests with non-positive profit due to Line 4, thus the optimum with respect to $\left(\mathcal{R}, p_{2}\right)$ is not greater than the optimum with respect to $\left(\mathcal{R}^{+}, p_{2}\right)$. It follows that $\mathcal{S}^{+}$is $\left(5+\frac{2}{1 / \rho-1}\right)$-approximate with respect to $\left(\mathcal{R}, p_{2}\right)$. Due to Line $3, p_{1}(Q)=0$, therefore adding

$Q$ to $\mathcal{S}$ (Line 6 ) does not change the profit of the solution. Hence, $\mathcal{S}$ is $\left(5+\frac{2}{1 / \rho-1}\right)$-approximate with respect to $\left(\mathcal{R}, p_{2}\right)$.

\section{2 $\quad$ Large Interferences}

In this section we present a $(2\lceil 1 / \rho\rceil+1)$-approximation algorithm for the case where all interferences are greater that $\rho$, namely for the case where $w(i)>\rho$, for every base station $i$.

Our algorithm is based on the following simple observation:

Observation 3. If $w(i)>\rho$, for every base station $i$, then at most $\lceil 1 / \rho\rceil$ base stations can transmit using the same frequency $t$. 
Our algorithm for the large interferences case is very similar to Algorithm FA-Small. More specifically, if $d(Q) \leq \frac{1}{2} f$, we modify the definition of $p_{1}$ at Line 3 as follows:

$$
p_{1}(R)=p(Q) \cdot \begin{cases}1 & R \in \mathcal{U}(Q), \\ \frac{2 d(R)}{f} & \text { otherwise }\end{cases}
$$

If $d(Q)>\frac{1}{2} f$, then the algorithm returns a solution that consists of an arbitrary allocation of the maximum profit request. The modified algorithm is called FA-Large.

The following two lemmas are similar to Lemmas 1 and 2.

Lemma 4. $\operatorname{OPT}\left(p_{1}\right) \leq(2\lceil 1 / \rho\rceil+1) p_{1}(Q)$.

Proof. Let $\left(\mathcal{S}^{\prime}, \mathcal{A}^{\prime}\right)$ be a feasible solution. By Observation 3 we have that

$$
p_{1}^{t}(\mathcal{S}(t) \backslash \mathcal{U}(Q)) \leq\lceil 1 / \rho\rceil \cdot p_{1}(Q) \frac{2}{f},
$$

for every frequency $t$. Hence,

$$
p_{1}\left(\mathcal{S}^{\prime}\right)=p_{1}\left(\mathcal{S}^{\prime} \cap \mathcal{U}(Q)\right)+p_{1}\left(\mathcal{S}^{\prime} \backslash \mathcal{U}(Q)\right) \leq p_{1}(Q)+2\lceil 1 / \rho\rceil p_{1}(Q)=(2\lceil 1 / \rho\rceil+1) p_{1}(Q),
$$

as required.

Lemma 5. If $d(Q) \leq \frac{1}{2} f$, then $p_{1}(\mathcal{S}) \geq p_{1}(Q)$.

Proof. First, if $\mathcal{S} \cap \mathcal{U}(Q) \neq \emptyset$, then we are done. Otherwise, if a frequency $t$ is unavailable for $Q$, then $t$ is assigned to at least one request. It follows that $p_{1}^{t}(\mathcal{S}(t) \backslash \mathcal{U}(Q)) \geq p_{1}(Q) \frac{2}{f}$. Since $d(Q) \leq \frac{1}{2} f$, we have that $p_{1}(\mathcal{S})=\sum_{t} p_{1}^{t}(\mathcal{S})>\frac{1}{2} f \cdot p_{1}(Q) \frac{2}{f}=p_{1}(Q)$.

It remains to prove that Algorithm FA-Large computes $(2\lceil 1 / \rho\rceil+1)$-approximate solutions.

Theorem 3. Let $\rho \leq \frac{1}{2}$. Algorithm FA-Large is a polynomial time $(2\lceil 1 / \rho\rceil+1)$-approximation algorithm for the special case of FAMI, where $w(i)>\rho$, for every $i$.

Proof. The proof is by induction on the recursion. At the induction base either $\mathcal{R}=\emptyset$ and the algorithm returns an optimal solution, or $\mathcal{S}$ contains the maximum profit request. It follows that $\mathcal{S}$ is $2\lceil 1 / \rho\rceil$-approximate. The inductive step is similar to the step in the proof of Theorem 2 , where Lemmas 1 and 2 are replaced by Lemmas 4 and 5 .

\subsection{General Case}

We utilize both FA-Small and FA-Large to obtain a 12-approximation algorithm for FAMI.

Theorem 4. There exists a polynomial time 12-approximation algorithm for FAMI.

Proof. Set $\rho=\frac{1}{2}$. Given an set of requests $\mathcal{R}$, we partition $\mathcal{R}$ into two instances one containing requests regarding base stations with small interferences $\left(w(i) \leq \frac{1}{2}\right.$, for all $\left.i\right)$, and the other containing requests regarding base station with large interferences $\left(w(i)>\frac{1}{2}\right.$, for all $\left.i\right)$. We denote the corresponding sets of requests by $\mathcal{R}_{S}$ and $\mathcal{R}_{L}$, respectively. Due to Theorems 2 and 3 we can compute a 7 -approximate solution $\mathcal{S}_{S}$ with respect to $\mathcal{R}_{S}$ and a 5 -approximate solution $\mathcal{S}_{L}$ with respect to $\mathcal{R}_{L}$. Now, let $\mathcal{S}^{*}$ be an optimal solution with respect to $\mathcal{R}$. Either $p\left(\mathcal{S}^{*} \cap \mathcal{R}_{S}\right) \geq \frac{7}{12} \cdot p\left(\mathcal{S}^{*}\right)$ or $w\left(\mathcal{S}^{*} \cap \mathcal{R}_{L}\right) \geq \frac{5}{12} \cdot p\left(S^{*}\right)$. Hence, either $p\left(\mathcal{S}_{S}\right) \geq \frac{1}{7} \cdot \frac{7}{12} \cdot p\left(\mathcal{S}^{*}\right)=\frac{1}{12} \cdot p\left(\mathcal{S}^{*}\right)$ or $p\left(\mathcal{S}_{L}\right) \geq \frac{1}{5} \cdot \frac{5}{12} \cdot p\left(\mathcal{S}^{*}\right)=\frac{1}{12} \cdot p\left(\mathcal{S}^{*}\right)$. If follows that the solution of greater profit is a 12 -approximation for the original instance $\mathcal{R}$. 
As mentioned in Section 2.1 the linearization of the multiplicative model may cause accuracy problems. A feasible solution with respect to the multiplicative model may be infeasible with respect to its additive linearization, and vice versa due to "rounding" of additive interferences. Our local ratio algorithms apply to FAMI with multiplicative interferences as long as feasibility is determined using the multiplicative model and not the additive model (e.g., in Line 6 of Algorithm FA-Small). If feasibility is checked using linearized additive interferences, the computed solution might be infeasible, and we may lose considerable amount of profit by fixing it. On the other hand, by checking feasibility in the multiplicative model the approximation ratios may slightly increase due to rounding issues. This may occur because the lower bounds on $p_{1}(\mathcal{S})$ (Lemmas 2 and 5) may slightly decrease, while the upper bounds on $\mathrm{OPT}\left(p_{1}\right)$ (Lemmas 1 and 4 ) may slightly increase.

We also note that our upper bounds improve to $4+\frac{2}{1 / \rho-1}, 2\lceil 1 / \rho\rceil$, and 10 , in the special case of FAMI, in which each user has only one request.

\section{Interval Scheduling with Multiple Interferences}

In this section we present our approximation algorithm for ISMI. As in the previous section we start with the additive interference model. For $\rho \in\left(0, \frac{1}{2}\right]$, we present a local ratio $\left(3+\frac{1}{1 / \rho-1}\right)$ approximation algorithm for the case where all interferences are at most $\rho$. We also design a $(\lceil 1 / \rho\rceil+1)$-approximation algorithm for the case where all interferences are greater than $\rho$. By combining both algorithms for $\rho=\frac{1}{2}$ we obtain a 7-approximation algorithm for the general case. Our approximation algorithm can be used for ISMI with the multiplicative interference model. In this multiplicative case, the $\left(3+\frac{1}{1 / \rho-1}\right)$ ratio is obtained for the case where $\sigma(i) \leq 1-(1-\delta)^{\rho}$, for every base station $i \in B$.

\subsection{Small Interferences}

In this section we present a $\left(3+\frac{1}{1 / \rho-1}\right)$-approximation algorithm for the case where all interferences are bounded by $\rho$, i.e., for the case where $w(i) \leq \rho$, for every $i \in B$.

Before presenting the algorithm we need some notation and definitions. Let $I$ be a request for base station $i$ made by user $j$. We use $r(I)$ to denote the right endpoint of $I$. Let $\mathcal{U}(I) \subseteq \mathcal{R}$ denote the set of requests made by user $j$ and let $\mathcal{B}(I) \subseteq \mathcal{R}$ denote the set of requests from base station $i$ at $r(I)$, i.e., $\mathcal{U}(I) \triangleq \bigcup_{i^{\prime}} \mathcal{R}_{j i^{\prime}}$ and $\mathcal{B}(I) \triangleq\left\{J: r(I) \in J \in \bigcup_{j^{\prime}} \mathcal{R}_{j^{\prime} i}\right\}$. Also, let $\mathcal{T}(I)$ be the set of requests that contain frequency $r(I)$ and that are made by some user $j^{\prime} \neq j$ to a base station $i^{\prime} \neq i$.

Algorithm IS-Small (Algorithm 2) is our local ratio algorithm for ISMI with small interferences. It is recursive and works as follows. If there are no requests, then it returns and empty schedule. Otherwise, it chooses a request $I$ with the leftmost right end point, namely such that $r(I) \leq r\left(I^{\prime}\right)$, for every $I^{\prime} \in \mathcal{R}$. It constructs a new profit function $p_{1}$, and solves the problem recursively on $p_{2}=p-p_{1}$ and the set of requests with positive profit with respect to $p_{2}$ that is denoted by $\mathcal{R}^{+}$. Notice that $I \notin \mathcal{R}^{+}$, since $p_{2}(I)=0$. Then, it adds $I$ to the solution that was computed recursively, if feasibility is maintained.

Observe that the computed solution $\mathcal{S}$ is feasible by construction. As for the running time, first observe that the number of recursive calls is at most $|\mathcal{R}|$, since each recursive call removes at least one request. In a naive implementation each iteration requires $O(|\mathcal{R}|)$ operations (finding a request with leftmost right endpoint, subtracting profits, and computing $\mathcal{R}^{+}$). Also notice that Line 6 can 


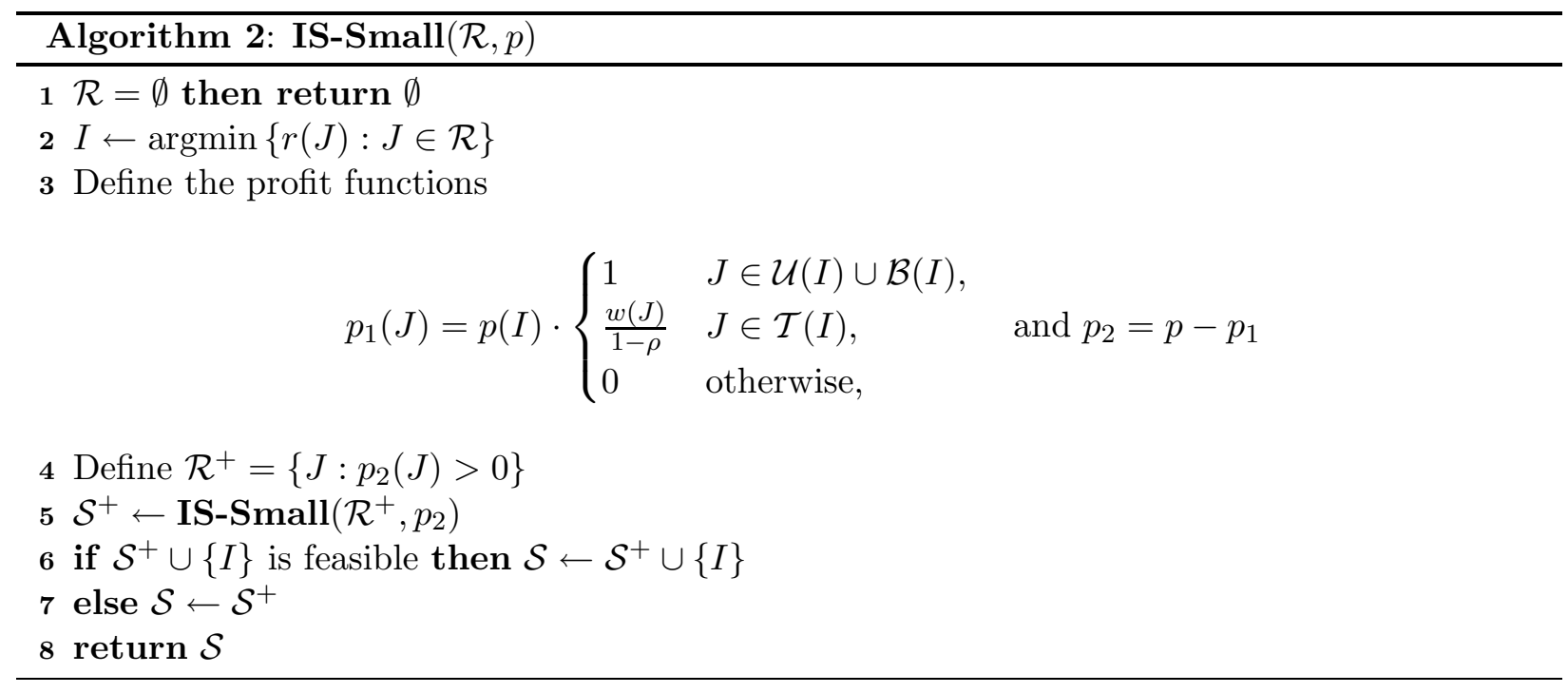

be implemented in $O(1)$, since we only need to check feasibility at $r(I)$. Hence, the running time of the algorithm is $O\left(|\mathcal{R}|^{2}\right)$.

We use the Local Ratio Theorem to prove that IS-Small is a $\left(3+\frac{1}{1 / \rho-1}\right)$-approximation algorithm. We start by showing that the profit of any feasible solution is at most $\left(3+\frac{1}{1 / \rho-1}\right) p_{1}(I)$ with respect to $p_{1}$.

Lemma 6. If $\rho \leq \frac{1}{2}$, then $\operatorname{OPT}\left(p_{1}\right) \leq\left(3+\frac{1}{1 / \rho-1}\right) p_{1}(I)$.

Proof. Let $i$ and $j$ be the base station and user that correspond to $I$, i.e., $I \in \mathcal{R}_{j i}$. By construction of $p_{1}$ the requests in $\mathcal{U}(I) \cup \mathcal{B}(I)$ are given profit $p_{1}(I)=p(I)$ (Line 3). Also, a request $J \in \mathcal{T}(I)$ is given profit $p_{1}(J)=p_{1}(I) \cdot \frac{w(J)}{1-\rho}$. All other requests have zero profit and therefore do not contribute to the profit of the solution with respect to $p_{1}$. Let $\mathcal{S}^{\prime}$ be a feasible solution. Since $\mathcal{S}^{\prime}$ may contain at most one request from $\mathcal{U}(I)$ (user constraint), it follows that $p_{1}\left(\mathcal{S}^{\prime} \cap \mathcal{U}(I)\right) \leq p_{1}(I)$. Furthermore, $\mathcal{S}^{\prime}$ may contain at most one request from $\mathcal{B}(I)$ (base station constraint). If $\mathcal{S}^{\prime}$ contains a request from $\mathcal{B}(I)$, then the overall interference from requests in $\mathcal{S}^{\prime} \cap \mathcal{T}(I)$ is at most 1 , hence $p_{1}\left(\mathcal{S}^{\prime} \cap \mathcal{T}(I)\right) \leq \frac{1}{1-\rho} p_{1}(I)$, and we have

$p_{1}\left(\mathcal{S}^{\prime}\right)=p_{1}\left(\mathcal{S}^{\prime} \cap \mathcal{U}(I)\right)+p_{1}\left(\mathcal{S}^{\prime} \cap \mathcal{B}(I)\right)+p_{1}\left(\mathcal{S}^{\prime} \cap \mathcal{T}(I)\right) \leq 2 p_{1}(I)+\frac{p_{1}(I)}{1-\rho}=\left(3+\frac{1}{1 / \rho-1}\right) p_{1}(I)$.

Otherwise, if $\mathcal{S}^{\prime} \cap \mathcal{B}(I)=\emptyset$, then $p_{1}\left(\mathcal{S}^{\prime} \cap \mathcal{T}(I)\right) \leq \frac{1+\rho}{1-\rho} \cdot p_{1}(I)=p_{1}(I)$, and therefore

$$
\begin{aligned}
p_{1}\left(\mathcal{S}^{\prime}\right) & =p_{1}\left(\mathcal{S}^{\prime} \cap \mathcal{U}(I)\right)+p_{1}\left(\mathcal{S}^{\prime} \cap \mathcal{T}(I)\right) \\
& \leq p_{1}(I)+\frac{1+\rho}{1-\rho} p_{1}(I) \\
& =\frac{2}{1-\rho} p_{1}(I) \\
& \leq\left(3+\frac{1}{1 / \rho-1}\right) p_{1}(I)
\end{aligned}
$$

where the last inequality follows from $\rho \leq \frac{1}{2}$. 
Next, we show that the profit of the computed solution $\mathcal{S}$ is at least $p_{1}(I)$ with respect to $p_{1}$.

Lemma 7. $p_{1}(\mathcal{S}) \geq p_{1}(I)$.

Proof. Due to Line 6 we know that either $I \in \mathcal{S}$ or $\mathcal{S} \cup\{I\}$ is infeasible. If $\mathcal{S} \cap(\mathcal{U}(I) \cup \mathcal{B}(I)) \neq \emptyset$, then $p_{1}(\mathcal{S}) \geq p_{1}(I)$. Otherwise, $I$ was rejected due to some interference constraint. Since $r(I)$ is the leftmost right endpoint in $\mathcal{R}$, any interference felt at frequency $t<r(I)$, is also felt at $r(I)$. Hence, $I$ was rejected due to the interference constraint at $r(I)$. In this case there is a request $I^{\prime} \in \mathcal{S} \cap \mathcal{T}(I) \cup\{I\}$ such that $w\left(\mathcal{S} \cap \mathcal{T}(I) \cup\{I\} \backslash\left\{I^{\prime}\right\}\right)>1$. Hence, $w(\mathcal{S} \cap \mathcal{T}(I))>1-\rho$ and thus $p_{1}(\mathcal{S} \cap \mathcal{T}(I)) \geq p_{1}(I)$.

The proof of the next theorem is similar to the proof of Theorem 2 where Lemmas 6 and 7 replace Lemmas 1 and 2 .

Theorem 5. Algorithm IS-Small is a polynomial time $\left(3+\frac{1}{1 / \rho-1}\right)$-approximation algorithm for the special case of ISMI, where $w(i) \leq \rho$, for every base station $i$.

\subsection{Large Interferences}

In this section we present a $(\lceil 1 / \rho\rceil+1)$-approximation algorithm for the case where all interferences are greater that $\rho$, namely for the case where $w(i)>\rho$, for every base station $i$.

Our algorithm for the large interferences case is very similar to Algorithm IS-Small. More specifically, we just modify the definition of $p_{1}$ at Line 3 as follows:

$$
p_{1}(J)=p(I) \cdot \begin{cases}1 & J \in \mathcal{U}(I) \cup \mathcal{B}(I) \cup \mathcal{T}(I), \\ 0 & \text { otherwise }\end{cases}
$$

The modified algorithm is called Algorithm IS-Large.

The following two lemmas are similar to Lemmas 6 and 7 .

Lemma 8. $\operatorname{OPT}\left(p_{1}\right) \leq(\lceil 1 / \rho\rceil+1) p_{1}(I)$.

Proof. Let $\mathcal{S}^{\prime}$ be a feasible solution. By Observation 3 we have that

$p_{1}\left(\mathcal{S}^{\prime}\right)=p_{1}\left(\mathcal{S}^{\prime} \cap \mathcal{U}(I)\right)+p_{1}\left(\mathcal{S}^{\prime} \cap \mathcal{B}(I)\right)+p_{1}\left(\mathcal{S}^{\prime} \cap \mathcal{T}(I)\right) \leq p_{1}(I)+\lceil 1 / \rho\rceil p_{1}(I)=(1+\lceil 1 / \rho\rceil) p_{1}(I)$,

and we are done.

Lemma 9. $p_{1}(\mathcal{S}) \geq p_{1}(I)$.

Proof. Line 6 implies that $\mathcal{S} \cap(\mathcal{U}(I) \cup \mathcal{B}(I) \cup \mathcal{T}(I)) \neq \emptyset$, and we are done.

The proof of the next theorem is similar to the proof of Theorem 2 .

Theorem 6. Algorithm IS-Large is a polynomial time $(\lceil 1 / \rho\rceil+1)$-approximation algorithm for the special case of ISMI, where $w(i)>\rho$, for every base station $i$. 


\subsection{General Case and Frequency Windows}

The following result can be obtained using similar arguments as in the proof of Theorem $4\left(\rho=\frac{1}{2}\right)$.

Theorem 7. There exists a polynomial time 7-approximation algorithm for ISMI.

The above result applies to ISMI with multiplicative interferences as long as feasibility is determined using the multiplicative model and not the additive model (e.g., in Line 6 of Algorithm ISSmall). This way the approximation ratios may slightly increase due to rounding issues.

We also note that our upper bounds improve to $2+\frac{1}{1 / \rho-1},\lceil 1 / \rho\rceil$, and 5 , in the special case of ISMI in which each user has only one request.

In ISMI with frequency windows each user $j \in U$ has $n$ sets $\mathcal{R}_{j 1}, \ldots, \mathcal{R}_{j n}$ of requests from base stations, where $\mathcal{R}_{j i}$ corresponds to base station $i \in B$. A request $R_{j i q} \in \mathcal{R}_{j i}$ consists of a frequency interval $\left[s_{j i q}, e_{j i q}\right]$, and a demand $d_{j i q}$, such that $s_{j i q}-e_{j i q} \geq d_{j i q}$. A profit $p\left(R_{j i q}\right)$ may be gained by assigning $j$ to any interval $\left[x, x+d_{j i q}\right)$ that is contained in $\left[s_{j i q}, e_{j i q}\right]$. A naive reduction to ISMI would be to transform each request with a frequency window to a set of requests. However, such a reduction may increase the number of requests by a factor of $\Theta(f)$. Instead we may use the approach of Bar-Noy et al. [6] that considered time windows in the context of JISP. Their approach can be used to obtain a $(7+\varepsilon)$-approximation algorithm for ISMI with frequency windows. Roughly speaking, this approach works as follows. When the input is in the form of frequency windows, we do not modify the profit of individual intervals, but rather operate on whole windows at a time. At each recursive call we find an interval $I$ with earliest end-time by considering $s_{j i q}+d_{j i q}$ for all remaining time windows. If $I$ 's profit is less than an $\varepsilon$-fraction of its original profit, then it is deleted. Otherwise, we update the profits of time windows according to the weight function $w_{1}$. This may cause time windows to split. Finally, we push $I$ on the stack. It can be shown that the number of time windows ever created is polynomial in $|\mathcal{R}|$ and $\frac{1}{\varepsilon}$ and that the running time is polynomial. The rest of details are similar to those given in [6] and therefore omitted.

\section{$5 \quad$ Hardness Results}

This section contains our hardness results. First we show that both FAMI and ISMI become very hard to approximate when the interference to a user listening to base station $i$ depends on both the interfering base station and on $i$. We also show that FAMI is strongly NP-hard in both interference models using a reduction from BIN PACKING. The same reduction applies to ISMI. However, recall that ISMI is APX-hard, since JISP is a special case of ISMI.

\subsection{Hardness of Pairwise Interference Model}

In this section we show that FAMI and ISMI become very hard to approximate if the interferences depend on both the interfered and the interfering base stations. The hardness result is given for FAMI, but since our reduction uses instances with a single frequency, the same result holds for ISMI, as well.

In the pairwise multiplicative interference model each pair of base stations $i$ and $k$ is associated with an interference parameter $\sigma(i, k)$ which represents the fraction of service that is lost by a user listening to $i$ while $k$ is transmitting. If several base stations are transmitting at frequency $t$, then the remaining service received by a user listening to base station $i$ at frequency $t$ is 
$\prod_{k \in B(t) \backslash\{i\}}(1-\sigma(i, k))$, where $B(t)$ denotes the set of base stations transmitting at $t$. A user is able to listen to base station $i$ at $t$ only if the interference at $t$ is at most $\delta$, i.e., if

$$
\prod_{k \in B(t) \backslash\{i\}}(1-\sigma(i, k)) \geq 1-\delta .
$$

Lund and Yannakakis [17] proved that, given a graph $G=(V, E)$, the problem of finding a maximum cardinality induced subgraph with maximum degree $\Delta$ is not approximable within $|V|^{\varepsilon}$, for some $\varepsilon>0$, unless $\mathrm{P}=\mathrm{NP}$. We show that FAMI is very hard to approximate using an approximation preserving reduction to this problem.

Theorem 8. FAMI with pairwise multiplicative interferences is not approximable within $n^{\varepsilon}$, for some $\varepsilon>0$, unless $P=N P$ even for the following special case:

1. $\sigma\left(i, i^{\prime}\right)=\sigma\left(i^{\prime}, i\right)$, for every $i$ and $i^{\prime}$.

2. $\log _{1-\delta}\left(1-\sigma\left(i, i^{\prime}\right)\right) \leq \frac{1}{k}$, for some constant $k \in \mathbb{N}$, and for every $i$ and $i^{\prime}$.

3. there is only one frequency.

4. at most one request per user.

5. at most one user per base station.

6. unit profits.

Proof. Given a graph $G=(V, E)$ we construct the following FAMI instance. There are $n=|V|$ base stations, and $n$ users and only one frequency $t_{0}$. User $i$ has only one request, denote by $R(i)$, for $t_{0}$ from base station $i$ whose profit is 1 . The interferences are defined according to the edges:

$$
\sigma\left(i, i^{\prime}\right)= \begin{cases}\frac{1}{2} & \left(i, i^{\prime}\right) \in E \\ 0 & \left(i, i^{\prime}\right) \notin E\end{cases}
$$

and finally $\delta=1-\frac{1}{2^{k}}$.

Let $V^{\prime}$ be a vertex set, and let $\mathcal{R}=\left\{R(i): i \in V^{\prime}\right\}$ be the corresponding set of requests. $V^{\prime}$ is feasible for $\Delta=k$ if and only is $\mathcal{R}$ is feasible, since

$$
\prod_{i^{\prime} \in V^{\prime} \backslash\{i\}}\left(1-\sigma\left(i, i^{\prime}\right)\right)=\prod_{i^{\prime} \in V^{\prime} \cap \Gamma(i)} \frac{1}{2}=\frac{1}{2^{\operatorname{deg}^{\prime}(i)}},
$$

for every $i \in V^{\prime}$, where $\Gamma(i)$ is the neighborhood of $i$ in $G$, and $\operatorname{deg}^{\prime}(i)=\left|V^{\prime} \cap \Gamma(i)\right|$.

Similar arguments can be used in the additive interference case.

Theorem 9. FAMI with pairwise additive interferences is not approximable within $n^{\varepsilon}$, for some $\varepsilon>0$, unless $P=N P$ even for the following special case

1. $w\left(i, i^{\prime}\right)=w\left(i^{\prime}, i\right)$, for every $i$ and $i^{\prime}$.

2. $w\left(i, i^{\prime}\right) \leq \frac{1}{k}$, for some constant $k \in \mathbb{N}$, and for every $i$ and $i^{\prime}$.

3. there is only one frequency.

4. at most one request per user.

5. at most one user per base station.

6. unit profits. 


\subsection{Hardness of FAMI}

In this section we show that FAMI is strongly NP-hard using a reduction from BIN PACKING.

Theorem 10. FAMI with both additive and multiplicative interferences is strongly NP-hard.

Proof. Assume we are given a BIN PACKING instance consisting of $m$ items of sizes $\alpha_{1}, \ldots, \alpha_{m} \in \mathbb{N}$, a bin of size $\beta \in \mathbb{N}$ and an integer $k$. (Notice that we may assume without loss of generality that $k \leq m$.) We construct a FAMI instance consisting of $k$ base stations, $m$ users and $\beta$ frequencies. The additive (multiplicative) interference of each base station is $w(i)=0$ (respectively, $\sigma(i)=0)$. For each item $j$ we define a user $j$ with $k$ demands each for $\alpha_{i}$ frequencies, one for each base station. All demands have unit profit. It is not hard to verify that the items can be packed in $k$ bins if and only if there exists a FAMI solution of profit $m$.

\section{Single Frequency Case \& The Backpack Problem}

In this section we focus on the special case of FAMI (or ISMI) with additive interferences in which each user has only one request whose interval contains a specific frequency $t_{0}$. Since this special case has only one interference constraint and no user constraints, it helps to understand the additive interference model. We show that even this special case of FAMI in NP-hard and that it is equivalent to an interesting variant of KNAPSACK, we call BACKPACK, that admits an FPTAS.

\subsection{The Backpack Problem}

Let $U_{i}$ denote the set of users that have a request corresponding to base station $i$, namely $U_{i}=$ $\left\{j: \mathcal{R}_{j i} \neq \emptyset\right\}$, for every $i$. Observe that in the special case of ISMI, in which each user has only one request whose interval contains $t_{0}$, the collection $U_{1}, \ldots, U_{n}$ is a partition of the users, i.e. $\bigcup_{i} U_{i}=U$ and $U_{i} \cap U_{k}=\emptyset$, for every $i \neq k$. Also, observe that due to the base station constraints each base station can serve at most one user. Hence we may assume, without loss of generality, that at most one user request correspond to base station $i$ by only considering the user with maximum profit within the set $U_{i}$.

The resulting problem can be described in KNAPSACK terms. The BACKPACK problem is defined as follows. We are given $n$ items, where item $i \in\{1, \ldots, n\}$ has a size $s_{i}$ and a profit $p_{i}$. The goal is to find a maximum profit subset of items $S$ such that

$$
\sum_{k \in S \backslash\{i\}} s_{k} \leq 1,
$$

for every item $i \in S$. (Recall that in the KNAPSACK problem the requirement is that $\sum_{k \in S} s_{k} \leq 1$.) Each item corresponds to a base station, and the size of an item $i$ corresponds to the interference of base station $i$. The profit of an item $i$ is equal to the profit of the user request that involves base station $i$, if such a request exists. Otherwise the item profit is zero.

In the sequel we show that (i) BACKPACK is NP-hard, and (ii) BACKPACK has an FPTAS.

\subsection{NP-hardness}

We show that BACKPACK is NP-hard using a reduction from KNAPSACK. Given a KNAPSACK instance consisting of $n$ items with sizes $s_{1}, \ldots, s_{n}$ and profits $p_{1}, \ldots, p_{n}$, we construct a BACKPACK instance by adding a new item $n+1$ such that $s_{n+1}=0$ and $p_{n+1}=\sum_{i=1}^{n} p_{i}+1$. 
Lemma 10. Let $q \geq 1$. There exist an instance solution of profit $q$ if and only if there exists a BACKPACK solution of profit $q+p_{n+1}$.

Proof. Observe that a BACKPACK solution of profit greater than $p_{n+1}$ must contain item $n+1$. Furthermore, $S \subseteq\{1, \ldots, n\}$ is a feasible KNAPSACK solution if and only if $S \cup\{n+1\}$ is a feasible BACKPACK solution. The lemma follows.

The following hardness result is implied by Lemma 10 .

Theorem 11. BACKPACK is NP-hard.

\subsection{Fully Polynomial Time Approximation Scheme for Backpack}

In this section we present an FPTAS for BACKPACK that is based on the FPTAS for KNAPSACK (see, e.g. [5, Chapter 2]). That is, we present a pseudo-polynomial algorithm for BACKPACK that can be transformed into an FPTAS using standard techniques.

Assume that the items are ordered such that their sizes are non-increasing, namely $s_{i} \geq s_{k}$ if $i<k$. Our dynamic programming algorithm is based on the following simple observation:

Observation 11. Let $S \subseteq\{1, \ldots, k-1\}$ be a feasible BACKPACK solution. The set $S \cup\{k\}$ is feasible if and only if $\sum_{i \in S} s_{i} \leq 1$.

Proof. If $S \cup\{k\}$ is feasible, then $\sum_{i \in S} s_{i} \leq 1$ due to Eqn. (4). On the other hand, observe that $s_{i^{\prime}} \geq s_{k}$, for every $i^{\prime}<k$. Hence, Eqn. (4) is satisfied, since

$$
s_{k}+\sum_{i \in S \backslash\left\{i^{\prime}\right\}} s_{i} \leq \sum_{i \in S} s_{i},
$$

for every $i^{\prime} \in S$. It follows that $S \cup\{k\}$ is feasible.

The dynamic programming table $\Pi$ is described as follows. The table entry $\Pi(k, p)$ stands for the minimum total size of a feasible solution that is contained in $\{1, \ldots, k\}$ and whose profit is exactly $p$. If such a solution does not exist, then $\Pi(k, p)=\infty$. The size of the table is $n P$, where $P=\sum_{i} p(i)$. The optimum value is the maximum value $p$ for which the entry $\Pi(n, p)$ is finite.

We now describe the process of computing the table entries. In the base case we have

$$
\Pi(1, p)= \begin{cases}s_{1} & p=p(1) \\ \infty & \text { otherwise }\end{cases}
$$

Also we define $\Pi(k, 0)=0$ for every $k$. Next we show how to compute an entry $\Pi(k, p)$, for $k>1$, using the following recursive equation:

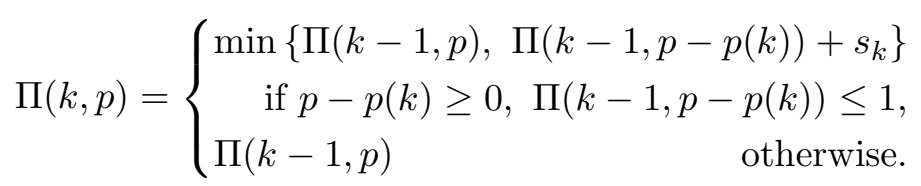

The idea is that if $\Pi(k-1, p-p(k)) \leq 1$, then by Observation 11 it is safe to add $k$ to the solution.

As for the running time, the initial sorting can be done in $O(n \log n)$ and computing an entry takes $O(1)$ time, thus the overall time complexity is $O(n P+n \log n)$. Also, note that the computation of $\Pi(k, p)$ can be modified to compute a corresponding optimal solution.

Using standard techniques we obtain the following result.

Theorem 12. There exists a fully polynomial time approximation scheme for BACKPACK. 


\section{Conclusion}

In this paper we have introduced the problem of bandwidth allocation with multiple interferences. We considered two variants of this problem. In the first variant each user request specifies a demand for a number of frequencies, while in the second each request is for a frequency interval. Furthermore, we considered two interference models, multiplicative and additive.

We showed that bandwidth allocation becomes extremely hard to approximate if the interferences depend on both the interfered and the interfering base stations, even for the special case where there is only one frequency. In light of this hardness result we considered the case where the interference depends only on the interfering base stations and provided constant factor approximation algorithms for the two variants of this problem. Our approximation algorithms use the uniformity of the interferences, namely they rely on the fact that the interference of a base station $i$ is the same for all users listening to any frequency assigned to a user request that is also used by $i$.

It would be very interesting to consider geometric pairwise interference models, such as the Signal to Interference and Noise Ratio (SINR) model that takes into account distances between users and base stations and the transmission power of interfered base stations. Also, it may be interesting to study frequency dependent interferences.

\section{References}

[1] D. Amzallag, R. Engelberg, J. Naor, and D. Raz. Coping with interference: From maximum coverage to planning cellular networks. Technical Report CS-2008-04, Computer Science Department, Technion, 2008.

[2] D. Amzallag, M. Livschitz, J. Naor, and D. Raz. Cell planning of $4 \mathrm{~g}$ cellular networks: Algorithmic techniques, and results. In 6th IEE International Conference on $3 G \& 3$ Beyond (3G'2005), pages 501$506,2005$.

[3] D. Amzallag, J. Naor, and D. Raz. Coping with interference: From maximum coverage to planning cellular networks. In 4th Workshop on Approximation and Online Algorithms, volume 4368 of LNCS, pages 29-42, 2006.

[4] D. Amzallag and D. Raz. Resource allocation problems for next generation cellular networks. In G. Cormode and M. Thottan, editors, Algorithms for Next Generation Networks, chapter 6, pages 99-129. Springer-Verlag, 2010.

[5] G. Ausiello, P. Creszenzi, G. Gambosi, V. Kann, A. Marchetti-Spaccamela, and M. Protasi. Complexity and approximation - Combinatorial optimization problems and their approximability properties. Springer-Verlag, 1999.

[6] A. Bar-Noy, R. Bar-Yehuda, A. Freund, J. Naor, and B. Shieber. A unified approach to approximating resource allocation and scheduling. J. ACM, 48(5):1069-1090, 2001.

[7] A. Bar-Noy, S. Guha, J. Naor, and B. Schieber. Approximating the throughput of multiple machines in real-time scheduling. SIAM J. Comput., 31(2):331-352, 2001.

[8] R. Bar-Yehuda and S. Even. A local-ratio theorem for approximating the weighted vertex cover problem. Annals of Discrete Mathematics, 25:27-46, 1985.

[9] M. F. Cátedra and J. Pérez-Arriaga. Cell Planning for Wireless Communications. Artech House, Inc., 1999. 
[10] J. Chuzhoy, R. Ostrovsky, and Y. Rabani. Approximation algorithms for the job interval selection problem and related scheduling problems. Math. Oper. Res., 31(4):730-738, 2006.

[11] F. C. Gomes, P. Pardalos, C. S. Oliveira, and M. G. C. Resende. Reactive GRASP with path relinking for channel assignment in mobile phone networks. In 5th international workshop on Discrete Algorithms and Methods for Mobile Computing and Communications (DIALM), pages 60-67, New York, NY, USA, 2001. ACM.

[12] O. H. Ibarra and C. E. Kim. Fast approximation algorithms for the knapsack and sum of subset problems. J. ACM, 22(4):463-468, 1975.

[13] R. M. Karp. Reducibility among combinatorial problems. In R. E. Miller and J. W. Thatcher, editors, Complexity of Computer Computations, pages 85-103, New York, 1972. Plenum Press.

[14] I. Katzela and M. Naghshineh. Channel assignment schemes for cellular mobile telecommunications: A comprehensive survey. IEEE Personal Communications, 3:10-31, 1996.

[15] R. Klasing, N. Morales, and S. Pérennes. On the complexity of bandwidth allocation in radio networks. Theor. Comput. Sci, 406(3):225-239, 2008.

[16] A. Koster. Frequency Assignment: Models and Algorithms. PhD thesis, Universiteit Maastricht, 1999.

[17] C. Lund and M. Yannakakis. The approximation of maximum subgraph problems. In 20th International Colloquium on Automata, Languages and Programming, volume 700 of LNCS, pages 40-51, 1993.

[18] R. A. Murphey, P. M. Pardalos, and M. G. Resende. Frequency assignment problems. In Handbook of Combinatorial Optimization, Supplement Volume A, chapter 6, pages 295-377. Kluwer Academic Publishers, 1999.

[19] S. Sahni. Approximate algorithms for the 0/1 knapsack problem. J. ACM, 22(1):115-124, 1975.

[20] F. C. R. Spieksma. On the approximability of an interval scheduling problem. J. Scheduling, 2:215-227, 1999.

[21] D. Tse and P. Viswanath. Fundamentals of Wireless Communication. Cambridge University Press, 2005. 